Hautarzt 2020 $\cdot 71: 542-552$

https://doi.org/10.1007/s00105-020-04610-6

Online publiziert: 20. Mai 2020

(c) Der/die Autor(en) 2020

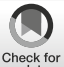

Eva Hadaschik' $\cdot$ Rüdiger Eming ${ }^{2}$ - Lars E. French ${ }^{3}$ - Giampiero Girolomoni ${ }^{4}$. Michael Hertl' ${ }^{2}$ Stephen Jolles ${ }^{5} \cdot$ Sarolta Karpati ${ }^{6} \cdot K^{2}$ Kerstin Steinbrink ${ }^{7}$. Georg Stingl $^{8} \cdot$ Beatrix Volc-Platzer $^{8} \cdot$ Detlef Zillikens $^{9} \cdot$ Alexander Enk $^{1}$

${ }^{1}$ Hautklinik, Ruprecht-Karls-Universität Heidelberg, Heidelberg, Deutschland

${ }^{2}$ Klinik für Dermatologie und Allergologie, Philipps-Universität Marburg, Marburg, Deutschland

${ }^{3}$ Dermatologische Klinik, Universität Zürich, Zürich, Schweiz

${ }^{4}$ Abteilung für Dermatologie, Universität Verona, Verona, Italien

${ }^{5}$ Immundefizienz-Zentrum für Wales, Universitätskrankenhaus Wales, Cardiff, Großbritannien

${ }^{6}$ Abteilung für Dermatologie, Semmelweis-Universität Budapest, Budapest, Ungarn

${ }^{7}$ Universitäts-Hautklinik, Johannes-Gutenberg-Universität Mainz, Mainz, Deutschland

${ }^{8}$ Universitätsklinik für Dermatologie, Medizinische Universität Wien, Wien, Österreich

${ }^{9}$ Klinik für Dermatologie, Allergologie und Venerologie, Universität zu Lübeck, Lübeck, Deutschland

\title{
Europäische Leitlinien (S1) für die Verwendung von hoch dosierten intravenösen Immunglobulinen in der Dermatologie
}

[CPMP]) der Europäischen Arzneimittelagentur (EMA) in Monographien der Europäischen Pharmakopöe veröffentlicht und laufend aktualisiert.

Folgende Schritte sind darin geregelt: Gewinnung des Plasmas, Überprüfungen des gespendeten Plasmas, Sicherheitsfragen im Zusammenhang mit Viren, Methoden der biologischen und pharmakologischen Charakterisierung und Tests der Endprodukte auf klinische Wirksamkeit. Für die Zulassung der Präparate sind die nationalen Behörden zuständig. In diesem Zusammenhang führen sie unter anderem Tests durch und legen fest, aus welchen Ländern Blut und Plasma bezogen werden darf. Die nationalen Behörden sind darüber hinaus für die regelmäßige Überprüfung des Herstellungsprozesses, der Durchführung virologischer Tests und für die Genehmigung von Änderungen im Herstellungsprozess verantwortlich.

Die Herstellung von Immunglobulinpräparaten beginnt mit der Identifizierung geeigneter Spender. Diese Spender müssen gesund sein und dürfen keine Anzeichen für Infektionen oder chronische Krankheiten aufweisen. Alle Plasmaspenden müssen frei von HBs-
Antigen und Anti-HCV-Antikörpern sowie negativ für HIV-1- und HIV-2Antikörper sein. Darüber hinaus unterliegen sämtliche Plasmaspenden einem Rückverfolgungsverfahren (Lookback) mit einer Quarantänedauer von mindestens 60 Tagen. Wird während dieses Zeitraums eine bei einem Spender aufgetretene Serokonversion erkannt, wird das gesamte unter Quarantäne gestellte Plasma von diesem Spender vernichtet. Die Überprüfung des Plasmas einzelner Spender oder des resultierenden Plasmagemisches auf HCV-RNA, HBV-DNA, HIV-RNA, HAV-RNA und Parvovirus-B19-DNA erfolgt mittels Techniken der Nukleinsäureamplifikation (NAT, Syn.: Polymerasekettenreaktion/PCR). Bei einem positiven Testergebnis werden die entsprechenden Plasmaspenden ausgemustert bzw. der Plasmapool wird vernichtet. Die Verarbeitung des Plasmas umfasst neben Schritten der Immunglobulinanreicherung verschiedene, voneinander unabhängige, Verfahrensschritte zur Inaktivierung oder Eliminierung von Viren. Zur Bewertung des Produktionsprozesses werden Testpräparationen mit einer Reihe Modellviren mit bzw. ohne Hülle versetzt, 


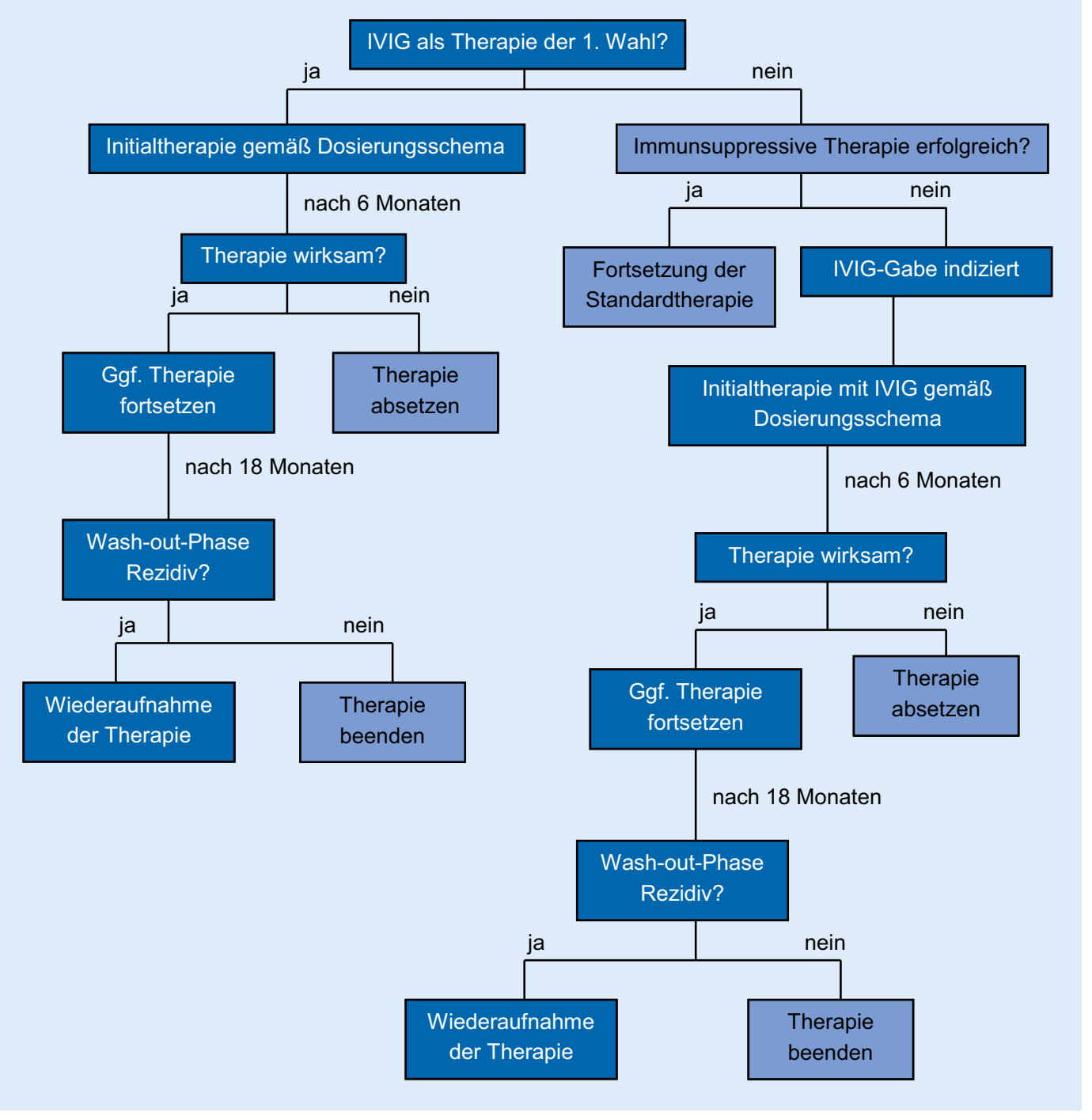

Abb. $1 \triangleleft$ Entscheidungsbaum für die Therapie mit IVIg

um die Reduktion der Viruslast bei jedem einzelnen Schritt des Prozesses zu quantifizieren und zu validieren.

Darüber hinaus kann es im Verarbeitungsprozess eine Reihe von speziellen Schritten zur Inaktivierung oder Eliminierung von Viren geben, die sich von Hersteller zu Hersteller unterscheiden. Für jede Charge eines produzierten Immunglobulinpräparates wird ein Zertifikat mit Informationen über die wichtigsten biologischen und pharmakologischen Eigenschaften, den Reinheitsgrad und das Antikörperspektrum erstellt.

Neben der Virussicherheit wird während des Herstellungsprozesses auch die klinische Wirksamkeit der Immunglobulinpräparate überprüft. Untersuchungen auf funktionelle Integrität, Bestimmung neutralisierender Antikörper und
Kontrolle immunmodulierender inflammatorischer Eigenschaften werden mit etablierten Methoden durchgeführt. Des Weiteren sind Studien bei Patienten mit einem primären Antikörpermangel vor der Zulassung erforderlich. Die erfolgreiche Behandlung von Patienten mit chronischer primärer Immunthrombozytopenie (ITP) wird als Nachweis der immunmodulierenden Aktivität eines Präparats erachtet.

Alle zurzeit kommerziell erhältlichen IVIg-Präparate bestehen aus intakten IgG-Molekülen mit einer IgGSubklassenverteilung, die dem normalen Antikörperspektrum entspricht. Die Halbwertszeit von IVIg bei gesunden Personen beträgt etwa 3 Wochen. Die Fc-Region des IgG ermöglicht die Interaktion und Signaltransduktion durch
Fc-gamma-Rezeptoren auf einer Reihe von Immunzellen. Die Wirkmechanismen der Immunglobuline sind komplex und in vivo noch nicht vollständig aufgeklärt. Das Verständnis der vielfältigen potenziellen Wirkmechanismen der Immunglobuline hat beträchtliche Fortschritte gemacht, und wahrscheinlich wirkt bei jeder einzelnen Erkrankung mehr als ein Mechanismus. Die Rolle von Fc-Rezeptoren wie der inhibitorische Rezeptor Fc $\gamma$ RIIB, die Effekte der Fc-Sialysierung sowie Änderungen bei regulatorischen T-Zellen (Treg) und des TH17-Signalweges fanden kürzlich Beachtung [1-4]. Immunglobuline werden seit mehr als 50 Jahren zur Behandlung von Krankheiten mit primärer oder sekundärer Immunschwäche eingesetzt. Die Nebenwirkungen der derzeitigen 
Produkte sind im Allgemeinen gering. Bei Hochdosistherapien sollten sich Ärzte jedoch bewusst sein, dass seltene schwere Nebenwirkungen wie thromboembolische Komplikationen auftreten können. In der Dermatologie werden IVIg hauptsächlich zur Behandlung von Autoimmunerkrankungen und toxischer epidermaler Nekrolyse eingesetzt [5]. Die Liste der behandelten Krankheiten ist zwar lang, basiert meist aber nur auf kleinen Fallserien oder einzelnen Fallberichten in unkontrollierten Studien. Dies erklärt sich zum Teil daraus, dass die Anzahl der Patienten mit diesen seltenen Erkrankungen für größere Studien zu klein ist und dass sich die Patienten wegen unterschiedlicher Verläufe und Begleitmedikationen nur schwer vergleichen lassen. Aufgrund der hohen Therapiekosten werden Immunglobulinpräparate äußerst selektiv eingesetzt, was die Untersuchung größerer Fallserien zusätzlich erschwert.

Das Ziel dieser Leitlinien ist es, für jede Erkrankung die folgenden Fragen zu beantworten:

1. Bei welchen Krankheiten sind IVIg angezeigt?

2. Sind IVIg als Erst- oder Zweitlinientherapie anzuwenden?

3. Wie lange dauert die Initialtherapie?

4. In welchen Zeitabständen sollten die IVIg-Infusionszyklen erfolgen?

5. Welche Immunglobulindosis sollte angewendet werden?

6. Wie lange dauert ein IVIg-Behandlungszyklus?

7. Gibt es Methoden zur Beurteilung der Wirksamkeit der Therapie?

8. Ist eine Langzeittherapie sinnvoll?

\section{Dermatomyositis}

Bei der Dermatomyositis liegt, abgesehen vom Pemphigus vulgaris, der höchste Evidenzgrad für eine Behandlung mit IVIg vor [6]. Es wurden zahlreiche einzelne Fallberichte und kleine Fallserien [7] sowie eine doppelblinde placebokontrollierte Crossover-Studie publiziert, die die Wirksamkeit von IVIg belegen [8]. Für die Anwendung von IVIg bei dieser Erkrankung wurden die folgenden Empfehlungen erarbeitet:

Hautarzt 2020 · 71:542-552 https://doi.org/10.1007/s00105-020-04610-6

(c) Der/die Autor(en) 2020

E. Hadaschik - R. Eming - L. E. French - G. Girolomoni - M. Hertl · S. Jolles · S. Karpati · K. Steinbrink - G. Stingl · B. Volc-Platzer · D. Zillikens · A. Enk

\section{Europäische Leitlinien (S1) für die Verwendung von hoch dosierten intravenösen Immunglobulinen in der Dermatologie}

Zusammenfassung

Hintergrund und Ziele. Die Behandlung schwerer dermatologischer Autoimmunerkrankungen und der toxischen epidermalen Nekrolyse (TEN) mit hoch dosierten intravenösen Immunglobulinen (IVIg) ist ein bewährtes therapeutisches Verfahren in der Dermatologie. Da eine IVIg-Therapie in der Regel nur bei seltenen Erkrankungen oder bei schweren Fällen in Betracht gezogen wird, stützt sich die Anwendung von Immunglobulinen zumeist nicht auf Daten aus randomisierten kontrollierten Studien, wie sie in der evidenzbasierten Medizin erforderlich sind. Da Indikationen für die Anwendung von IVIg selten sind, ist es unwahrscheinlich, dass solche Studien in absehbarer Zeit durchgeführt werden. Da der Einsatz von IVIg als Therapie der ersten Wahl aufgrund der hohen Kosten begrenzt ist, wurden erste klinische Leitlinien für die Anwendung von IVIg bei dermatologischen Erkrankungen im Jahr 2008 herausgegeben und im Jahr 2011 überarbeitet.

Patienten und Methoden. Die europäischen Leitlinien wurden von einer Gruppe durch das EDF („European Dermatology Forum") und die EADV („,European Academy of Dermatology and Venereology") benannter Experten erarbeitet. Die Leitlinien wurden erstellt, um die derzeit als wirksam erachteten Behandlungsindikationen zu aktualisieren und die Evidenz der für die Anwendung von IVIg bei dermatologischen Autoimmunerkrankungen und TEN vorliegenden Daten zusammenzufassen.

Ergebnisse und Schlussfolgerung. Die vorliegenden Leitlinien repräsentieren die einvernehmliche Meinung und Definitionen von Experten zur Anwendung von IVIg, die die aktuell publizierten Daten widerspiegeln, und sollen als Entscheidungshilfe für den Einsatz von IVIg bei dermatologischen Erkrankungen dienen.

\section{Schlüsselwörter}

Autoimmunerkrankungen · Dosierungen . Therapieschema · Expertenkonsens . Entscheidungsbaum

\section{European Guidelines (S1) on the use of high-dose intravenous immunoglobulin in dermatology}

Abstract

Background and objectives. Treatment of severe dermatological autoimmune diseases and toxic epidermal necrolysis (TEN) with high-dose intravenous immunoglobulin (IVIg) is a well-established procedure in dermatology. As treatment with IVIg is usually considered for rare clinical entities or severe cases, the use of immunoglobulin is not generally based on data from randomized controlled trials usually required for evidencebased medicine. Since the indications for the use of IVIg are rare, it is unlikely that such studies will be available in the foreseeable future. Because first-line use is limited by the high costs of IVIg, the first clinical guidelines on the use of IVIg in dermatological conditions were established in 2008 and renewed in 2011

Methods. The European guidelines presented here were prepared by a panel of experts nominated by the European Dermatology Forum (EDF) and European Academy of Dermatology and Venereology (EADV). The guidelines were developed to update the indications for treatment currently considered effective and to summarize the evidence for the use of IVIg in dermatological autoimmune diseases and TEN.

Results and conclusion. The current guidelines represent consensual expert opinions and definitions on the use of IVlg reflecting current published evidence and are intended to serve as a decision-making tool for the use of IVIg in dermatological diseases.

Keywords

Autoimmune diseases - Dosing - Therapy . Expert consensus · Decision tree 
1. Indikationen: Alle schweren Formen der Dermatomyositis, Einschlusskörpermyositis und Polymyositis stellen Indikationen für die Anwendung von IVIg dar [9]. Dies gilt jeweils auch für idiopathische, paraneoplastische oder juvenile Formen [10].

2. Zeitliche Planung der Behandlung: Die vorliegenden Daten rechtfertigen die frühe Anwendung von IVIg bei Dermatomyositis. Bei Patienten mit fulminantem Krankheitsverlauf, schwerer Myolyse oder Paralyse kann eine Erstlinientherapie mit Immunglobulinen vertretbar sein. In der Regel sollten IVIg als Zweitlinientherapie eingesetzt werden, wenn mit einer Steroidmonotherapie nach 1 Monat keine Verbesserung erzielt wurde, wenn die Reduzierung der Steroiddosis unter ein akzeptables Niveau zu einem Schub führt oder wenn eine weitere Steroidbehandlung aufgrund von Nebenwirkungen ausgeschlossen ist. Eine IVIg-Therapie wird als adjuvante Behandlung betrachtet, die in Kombination mit einer fortgesetzten immunsuppressiven Therapie mit Kortikosteroiden oder auch anderen immunsuppressiven Wirkstoffen durchgeführt wird [11]. Eine IVIg-Monotherapie ist im Allgemeinen weniger wirksam. Aus immunologischer Sicht muss für die gleichzeitige immunsuppressive Behandlung eine ausreichende Knochenmarkfunktion gewährleistet sein. Daher sollte mit der Einleitung der Behandlung nicht zu lange gewartet werden.

3. Dauer der Initialtherapie: Die Initialtherapie sollte sich über einen Zeittraum von 6 Monaten erstrecken, damit die Wirksamkeit der IVIgBehandlung beurteilt werden kann. Wurde nach 6 Behandlungszyklen keine therapeutische Wirkung erzielt, sollte die IVIg-Behandlung abgebrochen werden. Nach 18 Behandlungszyklen sollte versucht werden, eine Auswaschphase einzuschieben. Davor kann das Intervall zwischen den Infusionen auf maximal 6 Wochen verlängert werden (• Abb. 1). Bei Rezidiven kann die Behandlung jederzeit wieder aufgenommen wer- den. Diese Empfehlung ist an den individuellen Krankheitsverlauf anzupassen, da einige Patienten eine längere Therapie benötigen.

4. Intervall zwischen den Infusionen: Eingangs sollte eine adjuvante IVIgTherapie alle 4 Wochen verabreicht werden. Bei gutem klinischem Ansprechen kann das Intervall langsam bis auf maximal 6 Wochen verlängert werden. Längere Intervalle zwischen den Infusionen sind wegen der Halbwertszeit von IVIg (etwa 3 Wochen) nicht empfehlenswert.

5. Dosierung der IVIg: Die meisten Daten zur Anwendung von IVIg bei dermatologischen Autoimmunerkrankungen wurden mit einer Dosis von $2 \mathrm{~g}$ pro kg Körpergewicht pro Behandlungszyklus erhoben. Da es für die Wirksamkeit niedrigerer Dosen keine eindeutigen Belege gibt, ist es ratsam, sich bei diesen schweren Krankheiten an die oben gegebene Dosisempfehlung zu halten (• Tab. 1). Es gibt zwar einen Bericht über die erfolgreiche Behandlung von Polymyositis und Dermatomyositis durch subkutane Injektion von IgG bei 7 Patienten, jedoch muss diese Studie erst noch anhand größerer Patientenkohorten bestätigt werden [12]. Daher kann die subkutane Anwendung von IgG bei Dermatomyositis nicht generell empfohlen werden.

6. Dauer der IVIg-Behandlung: Die Immunglobulindosis sollte über einen Zeitraum von 2 bis 5 aufeinanderfolgenden Tagen verteilt werden. Im Allgemeinen ergibt sich bei Aufteilung der Gesamtdosis eine bessere Verträglichkeit. Bei Patienten mit Herz- oder Nierenfunktionsstörungen sollten Immunglobulinpräparate langsam verabreicht werden. Wird die Behandlung anfangs gut vertragen, kann sie auch ambulant durchgeführt werden.

7. Beurteilung der therapeutischen Wirksamkeit: Das klinische Bild ist bei der Behandlung der Dermatomyositis der wichtigste Parameter für die Bewertung der Wirksamkeit, wobei die Beurteilung der Muskelkraft die wichtigste Rolle spielt. Autoantikör- pertiter dagegen sind kein Maß für das Ansprechen auf die Behandlung. Im Allgemeinen normalisieren sich die Kreatinkinase- und Muskelaldolase-Spiegel unter immunsuppressiver Therapie. Daher eignen sich diese Parameter nicht als Indikatoren für die Wirksamkeit. Für die Erstdiagnose sind Magnetresonanztomographie oder Sonographie der proximalen Muskelgruppen ebenso wie Biopsien spezifischer Muskeln von Bedeutung. Für die engmaschige Kontrolle eignen sie sich jedoch nicht. Die Kriterien für die Beurteilung des klinischen Ansprechens sind daher die Normalisierung der Muskelkraft bei schrittweiser Verringerung der Steroiddosis, Abklingen des Erythems und allmähliche Rückbildung weiterer Symptome wie der Gottron-Papeln unter IVIg-Behandlung. Unserer Erfahrung nach kann ein Ansprechen ab dem zweiten Behandlungszyklus beobachtet werden, v. a. durch den Patienten (aufgrund der zunehmenden Muskelkraft), aber auch durch den behandelnden Arzt. Trotzdem sollte ein zu rasches Ausschleichen der Begleitmedikation vermieden werden. Häufig sind 3 bis 4 Behandlungszyklen nötig, bevor eine deutliche Verbesserung der Symptome erkennbar ist, und bei schwer betroffenen Patienten muss eine Verlängerung der Behandlungsintervalle mit Vorsicht erfolgen.

8. Langzeittherapie mit IVIg: In seltenen Fällen kann bei Patienten mit schwerer Dermatomyositis und langwierigem Verlauf eine Langzeittherapie erforderlich sein, wobei versuchsweise Therapieunterbrechungen eingeschoben werden sollten, um die Wirkung der IVIg-Behandlung auf den Krankheitsverlauf beurteilen zu können.

\section{Blasenbildende Autoimmun- erkrankungen}

Die blasenbildenden Autoimmundermatosen sind Autoantikörper-vermittelte Erkrankungen von Haut- und Schleimhäuten, deren auslösende Autoantigene weitgehend bekannt und moleku- 
Tab. 1 Hoch dosierte intravenöse Immunglobuline (IVlg): Dosierungsempfehlungen und The rapiedauer

\begin{tabular}{|c|c|}
\hline Dosis & Insgesamt $2 \mathrm{~g} / \mathrm{kg}$ Körpergewicht ${ }^{\mathrm{a}}$, verabreicht über 2 bis 5 Tage \\
\hline Behandlungsintervall & $\begin{array}{l}\text { Initial alle } 4 \text { Wochen; nach } 6 \text { Monaten stufenweise Verlängerung auf bis } \\
\text { zu } 6 \text { Wochen }^{\text {b }}\end{array}$ \\
\hline Langzeittherapie & In ausgewählten Fällen \\
\hline
\end{tabular}

lar charakterisiert sind. Blasenbildende Autoimmundermatosen werden nahezu ausschließlich von Dermatologen behandelt, weshalb sie für unser Fachgebiet von großer Bedeutung sind. Für die Anwendung von IVIg bei diesen Krankheiten wurden die folgenden Empfehlungen erarbeitet:

1. Indikation: Bei allen schweren Formen blasenbildender Autoimmundermatosen, die therapieresistent sind oder nach Therapie rezidivieren [13], sind IVIg indiziert. Eine randomisierte kontrollierte Studie [14] sowie eine gründliche Auswertung der Literatur [15] bestätigen diese Evidenzgrade. Es gibt besonders gute Erfahrungen in der Behandlung des Pemphigus vulgaris, Pemphigus foliaceus, Schleimhautpemphigoids [16] und der Epidermolysis bullosa acquisita [41]. Die Anwendung von IVIg kann jedoch auch bei schweren Formen des bullösen Pemphigoids [42], linearer IgA-Dermatose, IgAPemphigus oder paraneoplastischem Pemphigus angezeigt sein.

2. Zeitliche Planung der Behandlung: Auf der Grundlage der vorliegenden wissenschaftlichen Daten können IVIg nicht als Erstlinientherapie empfohlen werden. Bei Kontraindikationen für immunsuppressive Standardtherapien (beispielsweise aseptische Knochennekrose, schlecht kontrollierter Diabetes oder fortgeschrittene Osteoporose und Katarakt) kann jedoch in Einzelfällen der Einsatz von IVIg als Erstlinienbehandlung gerechtfertigt sein. Folglich sollten Immunglobuline hauptsächlich als Zweitlinienbehandlung nach einer ausreichenden Therapie mit Steroiden (beispielsweise Prednisolon 1-2 mg pro kg Körpergewicht pro Tag) und einem weiteren immunsup- pressiven Wirkstoff wie Azathioprin oder Mycophenolat-Mofetil angewendet werden $[17,18]$. Auch hier stellt die Behandlung mit IVIg eine adjuvante Therapie dar, die zusätzlich zu einer fortgesetzten herkömmlichen immunsuppressiven Therapie angewendet werden muss. Auch bei Patienten, die mit Rituximab behandelt wurden, ohne dass eine ausreichende Krankheitskontrolle erzielt wurde, können IVIg erwogen werden. Dies bedeutet auch, dass der Einsatz von Immunglobulinen nicht $\mathrm{zu}$ lange hinausgezögert werden sollte, da die adjuvante Behandlung nur bei gleichzeitiger immunsuppressiver Behandlung sinnvoll ist. Voraussetzung hierfür ist darüber hinaus eine ausreichende Knochenmarkfunktion. Eine Monotherapie mit Immunglobulinen wird im Allgemeinen nicht empfohlen.

3. Dauer der Initialtherapie: Die Initialtherapie sollte 3 bis 6 Monate dauern, damit die Wirksamkeit der IVIg im Einzelfall beurteilt werden kann. Bei einigen Patienten zeigt sich erst nach 6 Behandlungszyklen ein deutliches und anhaltendes Ansprechen. Ist nach 6 Behandlungszyklen kein therapeutischer Effekt zu beobachten, sollte die IVIg-Therapie abgebrochen werden (• Abb. 1). Diese Empfehlung ist bei jedem einzelnen Patienten an den Krankheitsverlauf anzupassen, da einige Patienten eine längere Therapie benötigen könnten.

4. Intervall zwischen den Infusionen: Die adjuvante Therapie mit IVIg sollte zu Beginn alle 4 Wochen verabreicht werden. Bei einem guten klinischen Ansprechen kann das Intervall zwischen den Infusionen nach und nach bis auf maximal 6 Wochen verlängert werden. Längere Intervalle sind wegen der Halbwertszeit der IVIg nicht empfehlenswert.

5. Dosierung: Wie oben bereits erwähnt, wurde bei den meisten Studien eine durch intravenöse Infusion verabreichte Gesamtdosis von $2 \mathrm{~g}$ pro kg Körpergewicht angewendet. Da bisher noch keine ausreichenden Daten zu höheren oder niedrigeren Dosen vorliegen, sollte diese Dosis zum gegenwärtigen Zeitpunkt als Standardempfehlung betrachtet werden (• Tab. 1).

6. Dauer der Behandlung: Wie bereits oben erwähnt, sollte die Dosis über einen Zeitraum von 2 bis 5 Tagen verabreicht werden, wobei eine Aufteilung der Dosis zu einer besseren Verträglichkeit beiträgt.

7. Beurteilung der therapeutischen Wirksamkeit: Bei den meisten blasenbildenden Autoimmundermatosen werden sowohl klinische als auch serologische Parameter herangezogen, um die Wirksamkeit der Behandlung zu beurteilen. Die Kriterien zur Bewertung des klinischen Bildes sind daher das Abklingen der Blasenbildung und das Abheilen vorhandener Läsionen unter adjuvanter IVIgTherapie. Gleichzeitig sollte eine moderate Reduzierung der begleitenden immunsuppressiven Behandlung ohne Rezidiv möglich sein. Zusätzlich können serologische Parameter, wie mittels ELISA bestimmte IgGAutoantikörpertiter, oder die indirekte Immunfluoreszenzmikroskopie zur Beurteilung der therapeutischen Wirksamkeit von IVIg herangezogen werden.

8. Langzeittherapie: Eine Langzeittherapie mit IVIg wird nur in seltenen Fällen empfohlen, insbesondere, wenn die Schleimhäute schwer betroffen sind. Eine Ausnahme bilden Patienten, bei denen die Erkrankung nach Absetzen der IVIg-Therapie rezidiviert und keine andere Behandlungsoption verfügbar ist. In diesem Fall kann eine Kombinationstherapie mit Rituximab erwogen werden. Es sollte regelmäßig versucht werden, Auswaschphasen einzuschieben. 


\section{Vaskulitiden}

Vaskulitiden sind systemische entzündliche Erkrankungen, die Blutgefäße in einem oder mehreren Organsystemen betreffen. Es wird zwischen primären und sekundären systemischen Vaskulitiden unterschieden. Da häufig die Haut als Indikatororgan mit betroffen ist und die Erkrankungen eine erhebliche Therapieresistenz zeigen, werden Immunglobuline oftmals als therapeutische Alternative angesehen. Auf der Grundlage des aktuellen Wissensstandes können folgende Empfehlungen gegeben werden:

1. Indikation: Das Kawasaki-Syndrom ist die einzige Erkrankung in dieser Kategorie, für die IVIg als Erstlinientherapie eingesetzt werden. In allen anderen Fällen erfolgt die Ersttherapie in der Regel mit hoch dosierten Kortikosteroiden zusammen mit weiteren immunsuppressiven Wirkstoffen wie Cyclophosphamid oder anderen. Die Anwendung dieser aggressiven immunsuppressiven Regime ist häufig mit schweren $\mathrm{Ne}$ benwirkungen verbunden, und bei Absetzen oder Dosisreduzierung kommt es zu Rezidiven. Bei Patienten, die auf eine Standardtherapie nicht ansprechen oder bei denen die Erkrankung fulminant progressiv verläuft, kann eine IVIg-Therapie als frühe Behandlungsoption erwogen werden. Alle schweren Vaskulitiden [19] können mögliche Indikationen für IVIg darstellen [20]. Besonders positive Ergebnisse wurden bei primärer Vaskulitis, wie z. B. Granulomatose mit Polyangiitis (WegenerGranulomatose), Polyarteriitis nodosa, IgA-assoziierter Vaskulitis, eosinophiler Granulomatose mit Polyangiitis (Churg-Strauss-Syndrom) [21], mikroskopischer Polyangiitis und sekundärer Autoimmunvaskulitis erzielt. Gute Ergebnisse zeigten sich auch bei Patienten mit Antiphospholipidsyndrom [22].

2. Zeitliche Planung der Behandlung: Zur Erstlinienbehandlung sind IVIg nur beim Kawasaki-Syndrom zugelassen. Wie bereits oben erwähnt, sollte die IVIg-Behandlung in allen anderen Indikationen als adjuvante Therapie nach Versagen einer immunsuppressiven Therapie oder bei Vorliegen von Kontraindikationen erwogen werden. Der frühe Einsatz von IVIg kann jedoch eine massive Zerstörung von Gewebe verhindern und so bei Erkrankungen, wie z. B. hämorrhagischer nekrotisierender Vaskulitis der Haut oder chronischer Polyangiitis, das Ausmaß der Schädigung verringern.

3. Dauer der Initialtherapie: Wie bei den bereits erwähnten Indikationen ist eine anfängliche Behandlungsdauer von 3 bis 6 Monaten sinnvoll, um sich ein klares Bild vom Ansprechen auf die Behandlung machen zu können (• Abb. 1).

4. Intervall zwischen den Infusionen: Wie oben dargestellt, sollte die Behandlung anfangs in vierwöchigen Intervallen erfolgen. Bei einem guten klinischen Ansprechen können die Intervalle zwischen den Infusionen nach und nach bis auf maximal 6 Wochen verlängert werden. Ein deutlicher Nutzen durch längere Behandlungsintervalle ist nicht dokumentiert.

5. Dosierung: Die empfohlene Dosis für die Behandlung des Kawasaki-Syndroms bei Kindern beträgt ebenfalls 1,6-2 g pro kg Körpergewicht pro Behandlungszyklus (als Bolusinfusion oder verteilt auf mehrere einzelne Infusionen über 2 bis 5 Tage) zusätzlich zur empfohlenen Gabe von Acetylsalicylsäure mit einer Anfangsdosis von $50 \mathrm{mg} / \mathrm{kg}$ Körpergewicht pro Tag. Entsprechend dem oben Gesagten wurden alle Fallserien von Patienten mit systemischen Vaskulitiden bisher mit einer Gesamtdosis von $2 \mathrm{~g}$ pro $\mathrm{kg}$ Körpergewicht behandelt (• Tab. 1).

6. Behandlungsdauer: Die Gesamtdosis sollte über einen Zeitraum von 2 bis 5 Tagen verabreicht werden, wobei ein längerer Zeitraum mit weniger Nebenwirkungen verbunden ist. Bei systemischer Vaskulitis, insbesondere mit Beteiligung der Nieren, sollte die Infusionsrate verringert oder möglicherweise eine niedrigere Dosis (beispielsweise insgesamt $1 \mathrm{~g}$ pro $\mathrm{kg}$ Körpergewicht) verwendet werden.
7. Beurteilung der therapeutischen Wirksamkeit: Das klinische Ansprechen sollte bei der Beurteilung der therapeutischen Wirksamkeit das Hauptkriterium darstellen. Da die verschiedenen Organe unterschiedlich stark betroffen sind, können nur allgemeine Empfehlungen gegeben werden. Das CRP-Muster und organspezifische Labortests können als Indikatoren für das Ansprechen genutzt werden. So können beispielsweise bei chronischer Polyangiitis der cANCA-Titer und der Proteinase3-Spiegel als zusätzliche Indikatoren dienen.

8. Langzeittherapie: Eine Langzeittherapie mit IVIg wird nur in Ausnahmefällen empfohlen.

\section{Systemischer Lupus ery- thematodes und andere Kollagenosen}

Fast alle autoimmunen Bindegewebserkrankungen wurden bereits im Rahmen kleiner Fallserien experimentell mit IVIg behandelt. Die aufschlussreichsten Daten liegen für den systemischen Lupus erythematodes vor. Folgende Empfehlungen werden gegeben:

1. Indikation: Alle schweren Fälle mit hämatologischen Komplikationen (Thrombopenie, autoimmunhämolytische Anämie) bei Patienten mit einem systemischen Lupus erythematodes können eine Indikation für die Behandlung mit IVIg darstellen, wenn keine anderen Behandlungsoptionen verfügbar sind. Über die hämatologischen Komplikationen hinaus wird IVIg insbesondere bei Lupusnephritis [23] als wirksam angesehen. Weniger klar sind die Daten bei Patienten mit Sklerodermie, für die keine klare Empfehlung gegeben werden kann [24]. Bei Bindegewebserkrankungen ist Vorsicht angeraten, da die Infusion von IVIg bei Patienten mit hohem Rheumafaktor(RF)-Titer zu Nierenschäden führen kann.

2. Zeitliche Planung der Behandlung: Die Anwendung von IVIg ist im Allgemeinen keine Option für eine Erstlinienbehandlung. Eine vorange- 
gangene Kombinationsbehandlung mit Steroiden und anderen Immunsuppressiva, die nicht zu einem zufriedenstellenden Ansprechen oder schweren Komplikationen führte, wird als Indikation für die Anwendung von IVIg betrachtet. Die Behandlung mit IVIg sollte jedoch auch bei Erkrankungen wie Lupusnephritis nicht zu lange hinausgeschoben werden, um Gewebeschädigung zu vermeiden. Auch hier sollte die Behandlung in Kombination mit einer geeigneten immunsuppressiven Therapie erfolgen.

3. Dauer der Initialtherapie: Wie bei den zuvor erwähnten Erkrankungen sollte die Anwendung von IVIg zunächst über einen Zeitraum von 6 Monaten erfolgen. Ist bis dahin kein Ansprechen erkennbar, sollte die Behandlung abgebrochen werden.

4. Intervall zwischen den Infusionen: Das Intervall zwischen den Infusionen sollte anfangs 4 Wochen betragen. Später kann das Intervall zwischen den einzelnen Bolusinfusionen allmählich auf 6 Wochen verlängert werden. Eine weitere Verlängerung des Intervalls ist aufgrund der Halbwertszeit der Immunglobuline nicht sinnvoll.

5. Dosierung: Auch bei diesen Störungen liegen nur für die Standarddosierung von $2 \mathrm{~g}$ pro kg Körpergewicht Erfahrungen vor. Diese Dosis sollte als Standardempfehlung übernommen werden (• Tab. 1).

6. Behandlungsdauer: Die IVIg-Dosis sollte über einen Zeitraum von 2 bis 5 Tagen verabreicht werden. Bei schwerer Organbeteiligung wie insbesondere der Nieren oder des Herzens sollte die Behandlungsdauer an 5 Tagen erfolgen.

7. Beurteilung der therapeutischen Wirksamkeit: Schwerpunkt ist einmal mehr die klinische Beurteilung der Wirksamkeit der Behandlung. Da es sich hier um eine sehr heterogene Gruppe von Krankheiten handelt, kann nur die allgemeine Empfehlung ausgesprochen werden, dass eine Verbesserung hinsichtlich des Befalls primärer Zielorgane (beispielsweise Proteinausscheidung mit dem Urin) als Indikator für ein Ansprechen verwendet werden sollte. In Einzelfällen kann das Muster krankheitsspezifischer Autoantikörper wie Antikörper gegen doppelsträngige DNA als ein Indikator für das Ansprechen bei Lupus erythematodes verwendet werden.

8. Langzeittherapie: Eine Langzeittherapie mit IVIg kann nur in Ausnahmefällen empfohlen werden.

\section{Skleromyxödem}

Das Skleromyxödem ist eine schwere Multiorganerkrankung, die durch Proliferation von Fibroblasten und $\mathrm{Mu}$ zinablagerung in der Haut und inneren Organen in Verbindung mit monoklonaler Gammopathie gekennzeichnet ist. Die Verdickung und Fibrosierung der Haut führen häufig zu einer starken Behinderung, und die Beteiligung innerer Organe bedeutet eine Gefahr für das Leben des Patienten [25, 26]. Das Skleromyxödem ist resistent gegenüber den meisten klassischen immunsuppressiven Therapien, spricht aber, wie in vielen Fallberichten und kleinen Fallserien dokumentiert, schnell auf die Behandlung mit IVIg an [27, 28]. Die Datenlage im Hinblick auf erfolgreiche Behandlungen des Skleromyxödems mit IVIg hat sich seit dem ersten Bericht im Jahr 2000 [26, 29] deutlich verbessert. Kürzlich zeigte eine prospektive, offene klinische Studie bei allen 8 Patienten mit Skleromyxödem eine signifikante klinische Besserung durch die IVIg-Behandlung [43]. Folgende Empfehlungen werden gegeben:

1. Indikation: Alle schweren Fälle von Skleromyxödem bilden eine Indikation für einen Therapieversuch mit IVIg, da die Behandlung mit anderen immunsuppressiven Wirkstoffen oft nicht wirksam ist. Die Anwendung von IVIg bei Skleromyxödem wird als wirksam betrachtet [26, 30, 31].

2. Zeitliche Planung der Behandlung: IVIg sollten bei therapieresistenten Fällen des Skleromyxödems mit schneller Verschlimmerung der Hautsymptome, mit DermatoNeuro-Syndrom oder bei lebensbedrohlicher Beteiligung von inneren
Organen als Therapie der Wahl angesehen werden. In leichteren Fällen sollte eine Initialbehandlung mit immunsuppressiven Regimen durchgeführt werden. Bei fehlendem Ansprechen auf diese Behandlung oder bei Kontraindikationen ist eine Therapie mit IVIg gerechtfertigt. Beim Skleromyxödem sind neben den IVIg keine zusätzlichen Behandlungen erforderlich.

3. Dauer der Initialtherapie: Wie bei den anderen erwähnten Erkrankungen sollte die Anwendung von IVIg zunächst über einen Zeitraum von 6 Monaten erfolgen. Lässt sich bis dahin kein Ansprechen erkennen, sollte die Behandlung abgebrochen werden.

4. Intervall zwischen den Infusionen: Das Intervall zwischen den Infusionen sollte auch hier zu Beginn 4 Wochen betragen. Später kann das Intervall zwischen den einzelnen Bolusinfusionen allmählich auf 6 Wochen verlängert werden. Eine weitere Verlängerung des Intervalls ist aufgrund der Halbwertszeit der Immunglobuline nicht sinnvoll.

5. Dosierung: Die meisten Erfahrungen zum Skleromyxödem liegen für die Standarddosis von $2 \mathrm{~g}$ pro kg Körpergewicht vor. Diese Dosis sollte als Standardempfehlung übernommen werden (• Tab. 1).

6. Behandlungsdauer: Die IVIg-Dosis sollte über einen Zeitraum von 2 bis 5 Tagen verabreicht werden. Bei schwerer Organbeteiligung, insbesondere der Nieren oder des Herzens, sollte die Behandlungsdauer 5 Tage betragen.

7. Beurteilung der therapeutischen Wirksamkeit: Der Schwerpunkt liegt einmal mehr auf der klinischen Beurteilung der Wirksamkeit der Behandlung. Da bei nahezu allen Fällen eine Beteiligung der Haut vorliegt, die auf die Behandlung mit IVIg gut anspricht, sollte diese als Indikator für das Ansprechen genutzt werden. In Einzelfällen kann das Ansprechen des ZNS oder von inneren Organen als zusätzlicher Indikator für das Ansprechen beim Skleromyxödem genutzt werden. 
Tab. 2 Dermatologische Erkrankungen,

bei denen die Anwendung von IVIg empfoh-

len wird

Indikationen für den Einsatz von IVIg

Schwere Formen der Dermatomyositis, Einschlusskörpermyositis, Polymyositis

Schwere Formen autoimmuner blasenbildender Erkrankungen

Schwere systemische Vaskulitiden

Systemischer Lupus erythematodes (hämatologische Komplikationen, schwere Lupusnephritis)

Skleromyxödem

Weniger eindeutige Indikationen

Toxische epidermale Nekrolyse

Autoimmune Urtikaria

Schwere Formen von Bindegewebserkrankungen

Livedovaskulopathie

8. Langzeittherapie: Es wurden einige Fälle dokumentiert, in denen es nach Abbruch der IVIg-Therapie zu Rezidiven kam [26, 32]. Ist ein Rezidiv schwer und lebensbedrohlich, kann in Ausnahmefällen eine Langzeittherapie empfohlen werden.

\section{Weitere mögliche Behandlungs- indikationen}

Die Behandlung mit IVIg wurde bei einer Vielzahl von dermatologischen Erkrankungen als wirksam beschrieben (- Tab. 2). Im Folgenden werden einige der häufigeren Entitäten erwähnt, wobei allerdings zurzeit eine abschließende Beurteilung nicht möglich ist.

\section{Toxische epidermale Nekrolyse}

Bei der toxischen epidermalen Nekrolyse handelt es sich um eine lebensbedrohliche Nebenwirkung von Arzneimitteln [33]. Die Erkrankung geht mit der Aktivierung der T-Zell-vermittelten Antwort (inklusive zytotoxisches Molekül Granulysin, Fas [CD95]-Ligand und Perforin/ Granzym B) und Apoptosemechanismen (inklusive miR-18a-5p-induzierte Apoptose, Annexine A1/Formyl-Peptidrezeptor-1-induzierte Nekroptose von Keratinozyten) einher [34]. In-vitroDaten zeigten, dass IVIg Antikörper enthalten, die Fas (CD95) binden und die Fas (CD95)-vermittelte Apoptose in Keratinozyten blockieren können [35].

Daher ist anzunehmen, dass Antikörper, die diese Apoptosesignaltransduktionswege stören (und in IVIg-Präparaten enthalten sind), bei diesem Krankheitsbild von Nutzen sind [35]. Aufgrund des lebensbedrohlichen und fulminanten progressiven Verlaufs der Erkrankung besteht bei diesen Patienten ein akutes Infektionsrisiko, und sie müssen intensivmedizinisch betreut werden. Für die Anwendung von IVIg wurden die folgenden Empfehlungen erarbeitet:

1. Indikation: In verschiedenen Fallserien ergab die frühzeitige Verabreichung hoher IVIg-Dosen bei toxischer epidermaler Nekrolyse eine signifikant niedrigere Mortalität im Vergleich zur der bei nicht behandelten Patienten mit SJS oder TEN. Bis heute wurden 5 Literaturanalysen von Fallkontrollstudien (Evidenzgrad 3a) durchgeführt, wobei nur bei 2 von ihnen der Effekt der Gesamt-IVIgDosis auf die Mortalität einbezogen wurde. Kein Review konnte einen Effekt von IVIg auf die Mortalität zeigen, wenn die zusammengefassten Patientendaten unabhängig von der verabreichten IVIg-Dosis betrachtet wurden. Jedoch zeigten 2 der Literaturanalysen, dass eine hoch dosierte IVIg-Therapie mit $\geq 2 \mathrm{~g} / \mathrm{kg}$ zu einer signifikant niedrigeren Mortalität führte als die Gabe von weniger als 2 g/kg IVIg [36, 37]. Obwohl die Wirkungsmechanismen noch unklar und die veröffentlichten Daten kontrovers sind und qualifizierte Studien mit hoch dosierten IVIg $(>2 \mathrm{~g} / \mathrm{kg})$ fehlen, kann die frühe Verabreichung hoch dosierter Immunglobuline bei bestätigten Fällen von toxischer epidermaler Nekrolyse erwogen werden. Insbesondere, wenn therapeutische Alternativen mit höherer Evidenz fehlen, überwiegt der angenommene mögliche Nutzen hoch dosierter IVIg die Risiken der Medikation und des natürlichen Verlaufs der Krankheit.

2. Zeitliche Planung der Behandlung: Anders als bei den zuvor erwähnten Erkrankungen sollten IVIg nach bestätigter Diagnose so früh wie möglich gegeben werden. Die IVIg-
Therapie kann als Monotherapie zusätzlich zu supportiven Maßnahmen einschließlich der intensivmedizinischen Behandlung verabreicht werden. Die gleichzeitige Gabe von Kortikosteroiden oder anderen immunsuppressiven Medikamenten wird kontrovers diskutiert.

3. Dauer der Initialtherapie: In der Regel ist bei dieser Erkrankung nur ein Behandlungszyklus erforderlich, der über einen Zeitraum von 3 bis 5 Tagen verabreicht wird.

4. Dosierung: Die für toxische epidermale Nekrolyse empfohlene Dosis unterscheidet sich von der bei anderen Autoimmunerkrankungen. Im Allgemeinen wird eine Gesamtdosis von mindestens $3 \mathrm{~g}$ pro kg Körpergewicht empfohlen (•Tab. 1). Eine fraktionierte Gabe über 3 bis 5 Tage ist erforderlich, insbesondere dann, wenn bei den Patienten Risikofaktoren wie eine Nierenfunktionsstörung, eine vorbestehende Herz-KreislaufErkrankung oder ein Diabetes vorliegen.

5. Beurteilung der therapeutischen Wirksamkeit: Das Sistieren der Epidermisablösung und eine einsetzende Reepithelisierung sind geeignete klinische Parameter zur Beurteilung der Wirksamkeit der Behandlung. Das beste Maß für den klinischen Therapieerfolg ist jedoch das Überleben. Der Beitrag von IVIg zum Heilungsprozess ist schwierig $\mathrm{zu}$ erfassen.

6. Langzeittherapie: Nicht zutreffend.

\section{Autoimmune Urtikaria}

Die Anwendung von Immunglobulinen kann bei schweren Fällen von autoimmuner Urtikaria als letzte Möglichkeit erwogen werden. Zurzeit liegen für diese Indikation nur einzelne Fallberichte und kleinere Fallserien vor, die den erfolgreichen Einsatz von Immunglobulinen in der oben erwähnten Standarddosierung beschreiben [38]. Eine endgültige Beurteilung dieser Berichte ist zum gegenwärtigen Zeitpunkt nicht möglich. In der Zukunft werden zunehmend neuere Therapien wie beispielsweise mit monoklonalen Anti-IgE-Antikörper bei thera- 
pieresistenter Urtikaria eine Rolle spielen.

\section{Pyoderma gangraenosum}

Pyoderma gangraenosum (PG) ist eine seltene neutrophile Dermatose, die sich klinisch mit Hautulzerationen aufgrund einer unerwünschten Aktivierung von Neutrophilen zeigt. Zunehmende Fallberichte und Fallserien berichten über den therapeutischen Effekt von IVIg bei schweren, therapierefraktären Patienten mit PG [44, 45]. In einer Übersicht wurde kürzlich beschrieben, dass von 49 mit IVIg behandelten PG-Patienten $88 \%$ ein partielles Ansprechen und $53 \%$ ein komplettes Ansprechen auf die Therapie zeigten. Aus diesem Grund kann die Anwendung von IVIg bei schweren therapieresistenten Fällen eines Pyoderma gangraenosum als Option erwogen werden, obwohl bislang keine allgemeine Konsensempfehlung vorliegt.

\section{Livedovaskulopathie}

Die Livedovaskulopathie (LV) ist klinisch durch wiederkehrende nekrotisierende Ulzerationen an den unteren Extremitäten aufgrund von Koagulationsstörungen charakterisiert.

In mehreren Fallstudien wird über einen erfolgreichen Einsatz von IVIg bei therapieresistenter LV berichtet $[39,40]$. Eine neuere Übersicht ergab ein hervorragendes Therapieergebnis bezüglich der Besserung von Schmerzen, Ulzerationen und Purpura bei 28 von 32 Patienten mit schwerer LV unter IVIG-Behandlung [46]. Obwohl zurzeit keine generellen Empfehlungen gegeben werden können, nehmen die Belege für einen positiven Effekt von IVIg zu, was ihren Einsatz in sehr schweren Fällen rechtfertigt.

\section{Zusammenfassung}

Die hier vorgelegten Behandlungsempfehlungen für die Anwendung von IVIg in der Dermatologie unterstreichen die Bedeutung der IVIg-Therapie bei zahlreichen definierten dermatologischen Autoimmunerkrankungen. Der Stellenwert der IVIg-Therapie bei ansonsten therapieresistenten Erkrankungen ist unbestritten. Für die oben aufgeführten Erkrankungen können daher Behandlungsempfehlungen gegeben werden. Da die exakten Wirkungsmechanismen von IVIg in vivo bei diesen Erkrankungen immer noch unklar sind, sollten weitere Anstrengungen unternommen werden, trotz der Seltenheit einiger der beschriebenen Erkrankungen randomisierte kontrollierte Studien durchzuführen. Die aktuellen Leitlinienempfehlungen sollen eine Grundlage für künftige randomisierte kontrollierte Studien bieten. Die Umsetzung dieser EU-Leitlinie in die allgemeine Praxis wird zu einer europaweiten Optimierung der Anwendung von IVIg in der Dermatologie führen.

\section{Methodik/weitere Informationen}

Die hier vorgelegten europäischen Leitlinien wurden von einer Gruppe durch das European Dermatology Forum (EDF) und die European Academy of Dermatology and Venereology (EADV) ernannter Experten erarbeitet, um die Indikationen vorzustellen, in denen eine Behandlung mit IVIg zurzeit als wirksam erachtet wird, und um die entsprechenden Belege zusammenzufassen.

Diese Leitlinien sollen es Dermatologen erleichtern, im Hinblick auf den Einsatz von IVIg fundierte therapeutische Entscheidungen zu treffen.

Die aktuellen Leitlinien repräsentieren die einvernehmlichen Meinungen und Definitionen von Experten zur Anwendung von IVIg, die die aktuell publizierten Daten widerspiegeln. Die Leitlinien wurden in 2 Runden der Evaluierung früherer Leitlinien (2017) erarbeitet, und zwar durch eine unabhängige Aktualisierung durch jedes der einzelnen repräsentativen Mitglieder der Expertengruppe, gefolgt von einem separaten Treffen aller Mitglieder mit Diskussion der festgelegten Themen. Die E-Mail-basierte Evaluierungsphase und das darauffolgende Treffen der Expertengruppe wurden von Professor Dr. med. A. Enk koordiniert und moderiert. Bei der Diskussion in der Gruppe wurde ein informeller Konsensus erzielt. Ein strukturiertes formelles Konsensusverfahren wurde nicht angewendet.
Das Leitlinienprojekt erhielt keine finanzielle Unterstützung. Die Expertengruppe erhielt keine finanziellen Anreize oder Erstattungen für die Beteiligung an der Entwicklung der Leitlinien. Die Zusammenfassung der Daten wurde unabhängig von industriellen Interessen vorgenommen.

Für die Teilnahme an diesem Projekt zur Leitlinienentwicklung war eine Erklärung zu möglichen Interessenkonflikten (COI) gemäß International Committee of Medical Journal Editors erforderlich. Die möglichen COI wurden diskutiert. Die Expertengruppe konnte keine wesentlichen Interessenkonflikte erkennen, und es gab keine weiteren Kommentare oder Bemerkungen. Die COI sämtlicher an der Leitlinienentwicklung beteiligter Personen sind im Anhang aufgeführt.

Die externe Überprüfung wurde der EDF-SOP für die Entwicklung von Leitlinien entsprechend durch Mitglieder des EDF-Leitlinienkomitees, des EADVVorstandes und der UEMS (Union Européenne des Médecins Spécialistes) über einen Zeitraum von 4 Wochen vorgenommen. Während der Überprüfungsphase wurde der Entwurf in den Abteilungen der teilnehmenden Experten in Umlauf gebracht. Kommentare und erforderliche Änderungen, die sich bei der externen Überprüfung ergeben hatten, wurden von den Autoren diskutiert. Die europäischen Leitlinien unterliegen der nationalen oder regionalen Anpassung unter Berücksichtigung der lokalen Situation (behördliche $\mathrm{Zu}$ lassung und Verfügbarkeit von Behandlungen, Gesundheitsdienstleister und Versicherungssysteme). Die dem EDF angeschlossenen nationalen medizinischen Gesellschaften sind verantwortlich für die Anpassung und Umsetzung der Leitlinien auf nationaler Ebene.

Aufgrund der ständig zunehmenden Anzahl an Publikationen müssen Leitlinien laufend aktualisiert werden, damit sie den jeweils aktuellen Wissensstand widerspiegeln. Nach dem 30.09.2022 verlieren die Leitlinien ihre Gültigkeit. Sollten sich in der Zwischenzeit wichtige Änderungen ergeben, wie die Verfügbarkeit neuer Interventionen, wichtiger neuer Daten oder die Rücknahme der Zulas- 
sung, sind die in den Leitlinien enthaltenen Informationen bereits früher veraltet. In diesem Fall ist eine aktualisierte Fassung der Leitlinien zu einem früheren Zeitpunkt nötig. Das EDF in Zusammenarbeit mit dem derzeitigen Leitlinienkoordinator ist für die Einleitung einer Aktualisierung verantwortlich.

\section{Korrespondenzadresse}

\section{Prof. Dr. med. Alexander Enk}

Hautklinik, Ruprecht-Karls-Universität

Heidelberg

Im Neuenheimer Feld 440, 69120 Heidelberg

Deutschland

alexander.enk@med.uni-heidelberg.de

Funding. Open Access funding provided by Projekt DEAL.

\section{Einhaltung ethischer Richtlinien}

Interessenkonflikt. E. Hadaschik hat Beraterhonorare von der Firma Biotest erhalten. A. Enk hat Beraterhonorare der Firmen Biotest, MSD, Galderma, BMS, Janssen, Lilly und Versabmed erhalten. D. Zillikens erhielt Beraterhonorare/Referentenhonorare der Firmen Fresenius, Roche, Janssen und Biotest. R. Eming, L.E. French, G. Girolomoni, M. Hertl, S. Jolles, S. Karpati, K. Steinbrink, G. Stingl und B. Volc-Platzer geben an, dass kein Interessenkonflikt besteht.

Für diesen Beitrag wurden von den Autoren keine Studien an Menschen oder Tieren durchgeführt. Für die aufgeführten Studien gelten die jeweils dort angegebenen ethischen Richtlinien.

Open Access. Dieser Artikel wird unter der Creative Commons Namensnennung 4.0 International Lizenz veröffentlicht, welche die Nutzung, Vervielfältigung, Bearbeitung, Verbreitung und Wiedergabe in jeglichem Medium und Format erlaubt, sofern Sie den/die ursprünglichen Autor(en) und die Quelle ordnungsgemäß nennen, einen Link zur Creative Commons Lizenz beifügen und angeben, ob Änderungen vorgenommen wurden.

Die in diesem Artikel enthaltenen Bilder und sonstiges Drittmaterial unterliegen ebenfalls der genannten Creative Commons Lizenz, sofern sich aus der Abbildungslegende nichts anderes ergibt. Sofern das betreffende Material nicht unter der genannten Creative Commons Lizenz steht und die betreffende Handlung nicht nach gesetzlichen Vorschriften erlaubt ist, ist für die oben aufgeführten Weiterverwendungen des Materials die Einwilligung des jeweiligen Rechteinhabers einzuholen.

Weitere Details zur Lizenz entnehmen Sie bitte der Lizenzinformation auf http://creativecommons.org/ licenses/by/4.0/deed.de.

\section{Literatur}

1. Schwab I, Nimmerjahn F (2013) Intravenous immunoglobulin therapy: how does IgG modulate theimmune system? Nat Rev Immunol 13:176-189

2. Anthony RM, Kobayashi T, Wermeling F et al (2011) Intravenous gammaglobulin suppresses inflammation through a novel $\mathrm{T}(\mathrm{H}) 2$ pathway. Nature 475:110-113

3. De Groot AS, Moise L, McMurray JA et al (2008) Activation of natural regulatory T cells by IgG Fc-derived peptide "Tregitopes". Blood 112:3303-3311

4. Maddur MS, Sharma M, Hedge $P$ et al (2013) Inhibitory effect of IVIG on IL17-production by Th17 cells is independent of anti-IL-17 antibodies in the immunoglobulin preparation. J Clin Immunol 33:62-66

5. Enk A, Hertl M, Messer G et al (2003) The use of high dose intravenous immunoglobulins in dermatology. J Dtsch Dermatol Ges 1:183-190

6. Jolles S, Hughes J, Whittaker S (1998) Dermatological uses of high-dose intravenous immunoglobulin. Arch Dermatol 134:80-86

7. van de Vlekkert J, Tjin-A-Ton ML, Hoogendijk JE (2004) Quality of myositis case reports open to improvement. Arthritis Rheum 51:148-150

8. Dalakas MC, Illa I, Dambrosia JM et al (1993) A controlled trial of high-dose intravenous immune infusions as treatment for dermatomyositis. NEng JMed 27:1993-2000

9. Gordon PA, Winer JB, Hoogendijk JE et al (2012) Immunosuppressant and immunomodulatory treatment for dermatomyositis and polymyositis. Cochrane Database Syst Rev 8:CD3643

10. Sansome A, Dubowitz V (1995) Intravenous immunoglobulin in juvenile dermatomyositis - fou year review of nine cases. Arch Dis Child 72:25-28

11. Dalakas MC (1995) Update on the use of intravenous immune globulin in the treatment of patients with inflammatory muscle disease. J Clin Immunol 15:70S-75S

12. Danieli MG, Pettinari L, Moretti R et al (2011) Subcutaneous immunoglobulin in polymyositis and dermatomyositis: a novel application. Autoimmun Rev 10:144-149

13. Murrell DF, DickS, Ahmed ARet al (2008) Consensus statement on definitions on disease, end points and therapeutic response for pemphigus. J Am Acad Dermatol 58:1043-1046

14. Amagai M, Ikeda S, Shimizu H et al (2009) A randomized double-blind trial of intravenous immunoglobulin for pemphigus. J Am Acad Dermatol 60:595-603

15. Gürcan HM, Jeph S, Ahmed AR (2010) Intravenous immunoglobulin therapy in autoimmune mucocutaneous blistering disease: a review of the evidence for its efficacy and safety. Am J Clin Dermatol 11:315-326

16. Letko $E$, Miserocchi $E$, Daoud $Y J$ et al (2004) A nonrandomized comparison of the clinical outcome of ocular involvement in patients with mucous membrane (cicatricial) pemphigoid between conventional immunosuppressive and intravenous immunoglobulin therapies. Clin Immunol 111:303-310

17. Hertl M, Jedlickova H, Karpati S et al (2015) Pemphigus. S2 guideline for diagnosis and treatment-guided by the European dermatology forum (EDF) in cooperation with the European academy of dermatology and venereology (EADV). JEur Acad Dermatol Venereol 29:405-415

18. Beissert S, Werfel T, Frieling U et al (2006) A comparison of oral methylprednisolone plus azathioprine or mycophenolate mofetil for the treatment of pemphigus. Arch Dermatol 142:1447-1454

19. Richter C, Schnabel A, Csernok E et al (1995) Treatment of anti-neutrophil cytoplasmic antibody (ANCA)-associated systemic vasculitis with high-dose intravenous immunoglobulin. Clin Exp Immunol 101:2-7

20. Aries PM, Hellmich B, Gross WL (2005) Intravenous immunoglobulin therapy in vasculitis. Clin Rev Allergy Immunol 29:237-245

21. Levy Y, George J, Fabbrizzi F et al (1999) Marked improvement of Churg-Strauss vasculitis with intravenous gammaglobulins. South Med J 92:412-414

22. Shoenfeld Y, Katz U (2005) IVlg therapy in autoimmunity and related disorders: our experience with a large cohort of patients. Autoimmunity 38:123-137

23. Toubi E, Kessel A, Shoenfeld Y (2005) High dose intravenous immunoglobulins: an option in the treatment of systemic lupus erythematosus. Hum Immunol 66:395-402

24. Levy Y, Langevitz P, Nacci F et al (2004) Intravenous immunoglobulin modulates cutaneous involvement and reduces skin fibrosis in systemic sclerosis: an open label study. Arthritis Rheum 50:1005-1007

25. Kukova G, Bruch-Gerharz D, Gensch K et al (2006) Skleromyxödem. Hautarzt 57:326

26. Rongioletti F, Merlo G, Cinotti E et al (2013) Scleromyxedema: a multicenter study of characteristics, comorbidities, course, and therapy in 30 patients. JAm Acad Dermatol 69:66-72

27. Karim A, Lawlor F, Black MM (2004) Successful treatment of scleromyxoedema with high dose intravenous immunoglobulin. Clin Exp Dermatol 29:317-318

28. Körber A, Franckson T, Grabbe S et al (2007) Successful therapy of scleromyxoedema ArndtGottron with low-dose intravenous immunoglobulin. J Eur Acad Dermatol Venereol 21:553-554

29. Lister RK, Jolles S, Whittaker S et al (2000) Scleromyxedema: response to high-dose intravenous immunoglobulin. J Am Acad Dermatol 43:403-408

30. Topf S, Jr. Simon M, Schell H et al (2007) Deutliche Besserung eines Skleromyxödem Arndt-Gottron durch hoch dosierte intravenöse Immunglobuline. Hautarzt 58:525-528

31. Blum M, Wigley FM, Hummers LK (2008) Scleromyxedema. A case series highlighting longterm outcomes of treatment with intravenous immunoglobulin (IVIG). Medicine 87:10-20

32. Bidier M, Zschoche C, Gholam P et al (2012) Scleromyxedema: clinical follow-up after successful treatment reveals different long-term outcomes. Acta Derm Venereol 92:408-409

33. Hötzenecker W, Prins C, French LE (2018) Erythema multiforme, Stevens-Johnson syndrome, and toxic epidermal necrolysis. In: Bolognia J, Schaffer J, Cerroni L (Hrsg) Dermatology, 4. Aufl. Elsevier, Amsterdam

34. Chung WH, Wang CW, Dao RL (2016) Severe cutaneous adverse drug reactions. J Dermatol 43:758-766

35. Viard I, Wehrli P, Bullani R et al (1998) Inhibition of toxic epidermal necrolysis by blockade of CD95 with human intravenous immunoglobulin. Science 282:490-493

36. Huang YC, Ly YC, Chen TC (2012) The efficacy of intravenous immunoglobulin for the treatment of toxicepidermal necrolysis: a systematic review and meta-analysis. Br J Dermatol 167:424-432 
37. Barron SJ, Del Vecchio LT, Aronoff SC (2015) Intravenous immunoglobulin in the treatment of Stevens-Johnson syndrome and toxic epidermal necrolysis:a meta-analysis with meta-regression of observational studies. Int J Dermatol 54:108-115

38. Jolles S, HughesJ (2006) Use of IGIVin the treatment of atopic dermatitis, urticaria, scleromyxedema, pyoderma gangrenosum, psoriasis, and pretibial myxedema. Int JImmunopharmacol 6:579-591

39. Schanz S, Ulmer A, Fierlbeck G (2003) Intravenous immunoglobulin in livedovasculitis: a new treatment option? J Am Acad Dermatol 49:555-556

40. Monshi B, Posch C, Vujic I et al (2014) Efficacy of intravenous immunoglobulins in livedoid vasculopathy: long-term follow-up of 11 patients. J Am Acad Dermatol 71:738-744

41. Iwata H, Vorobyev A, Koga $\mathrm{H}$ et al (2018) Metaanalysis of the clinical and immunopathological characteristics and treatment outcomes in epidermolysis bullosa acquisita patients. Orphanet JRare Dis 13(1):153

42. Amagai M, Ikeda S, Hashimoto T et al (2017) A randomized double-blind trial of intravenous immunoglobulin for bullous pemphigoid. JDermatol Sci 85(2):77-84

43. Guarneri A, Cioni M, Rongioletti F (2017) Highdose intravenous immunoglobulin therapy for scleromyxoedema: a prospective open-label clinical trial using an objective score of clinical evaluation system. J Eur Acad Dermatol Venereol 31(7):1157-1160

44. Song H, Lahood N, Mostaghimi A (2018) Intravenous immunoglobulin as adjunct therapy for refractory pyoderma gangrenosum: systematic review of cases and case series. Br J Dermatol 178(2):363-368

45. Herberger K, Dissemond J, Bruggestrat S, Sorbe C, Augustin M (2019) Biologics and immunoglobulins in the treatment of pyoderma gangrenosum - analysis of 52 patients. J Dtsch Dermatol Ges 17(1):32-41

46. Micieli R, Alavi A (2018) Treatment for livedoid vasculopathy: a systematic review. JAMA Dermatol 154(2):193-202

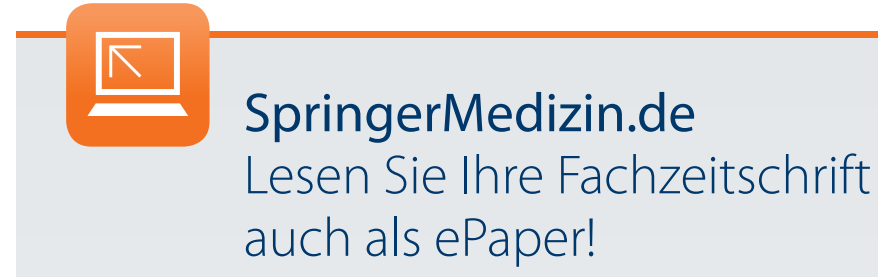

Als Abonnent können Sie Ihre Zeitschrift in verschiedenen Formaten lesen. Wählen Sie je nach Vorliebe und Situation aus, ob Sie die Zeitschrift als Print-Ausgabe, in Form von einzelnen Beiträgen auf springermedizin.de oder aber als komplette, elektronische ePaper-Ausgabe lesen möchten.

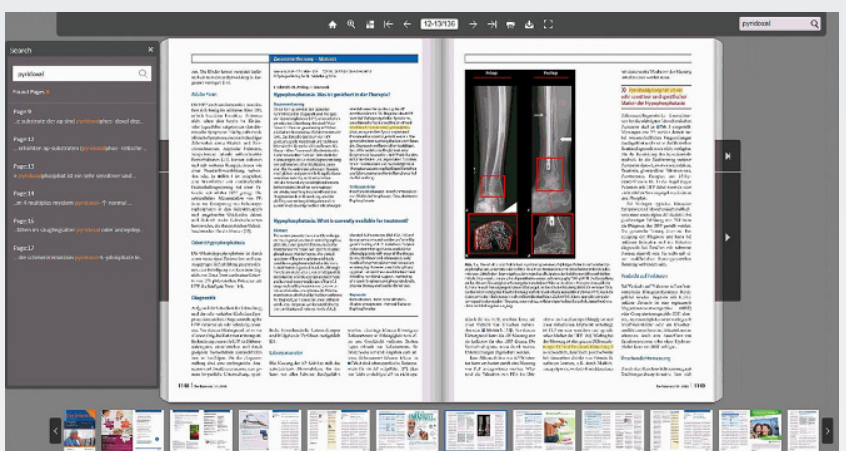

Die ePaper sind die identische Form der gedruckten Ausgaben. Sie sind nutzbar auf verschiedenen Endgeräten wie PC, Tablet oder Smartphone

Das sind die Vorteile des ePapers:

> Das verlinkte Inhaltsverzeichnis führt Sie direkt zum gewünschten Beitrag.

$>$ Eine Suchfunktion ermöglicht das Auffinden von Schlagworten innerhalb der Zeitschrift

$>$ Jede Ausgabe kann als PDF heruntergeladen und damit auch offline gelesen werden bzw. auch gespeichert oder ausgedruckt werden.

> Als Abonnent haben Sie Zugang zu allen ePaper-Ausgaben ab 2016.

Sie finden die ePaper auf SpringerMedizin.de bei der jeweiligen Ausgabe Ihrer

Fachzeitschrift. Klicken Sie auf den Button „Ausgabe als ePaper lesen”. 